\title{
Potential benefits and consequences of public reporting of pediatric cardiac surgery outcomes
}

\author{
J. William Gaynor, MD, ${ }^{a}$ Sara K. Pasquali, MD, MHS, ${ }^{b}$ Richard G. Ohye, MD, ${ }^{b}$ and Thomas L. Spray, MD $^{a}$
}

\footnotetext{
From the ${ }^{\mathrm{a} C a r d i a c}$ Center, The Children's Hospital of Philadelphia, Philadelphia, Pa; and ${ }^{\mathrm{b}}$ Congenital Heart Center, University of Michigan C.S. Mott Children's Hospital, Ann Arbor, Mich.

Received for publication July 30, 2016; revisions received Aug 26, 2016; accepted for publication Aug 26, 2016; available ahead of print Dec 2, 2016.

Address for reprints: J. William Gaynor, MD, Division of Cardiothoracic Surgery, The Children's Hospital of Philadelphia, 34th and Civic Center Blvd, Philadelphia, PA 19104 (E-mail: gaynor@email.chop.edu).

J Thorac Cardiovasc Surg 2017;153:904-7

$0022-5223 / \$ 36.00$

Copyright (C) 2016 Published by Elsevier Inc. on behalf of The American Association for Thoracic Surgery http://dx.doi.org/10.1016/j.jtcvs.2016.08.066
}

Feature Editor's Note-In this issue of The Journal, we focus on the evolving profile of public outcomes reporting initiatives in pediatric and congenital heart surgery. The dialogue around this topic has been intense and, at times, quite contentious. On more than one occasion, the debate has spilled over into the lay press and the court of public opinion, garnering focus and attention from the public media. Issues central to this debate include risk stratification methodologies, data accuracy, interpretation challenges, and potential negative consequences, including public criticism of individual surgeons and theoretical effects on patient access to timely surgery. Despite these and other concerns, the immutable reality is that the public has demanded and deserves access to objective information about performance in pediatric heart surgery. The challenge is how to move this process forward in a positive and professional supportive manner, while adhering to the ideal of transparency. To frame this issue, we have invited 2 groups to provide perspective within the context of a recent publication in the Journal of the American College of Cardiology by DeVore and colleagues.

\section{Charles Fraser, MD}

There is increasing demand across multiple stakeholders for transparency and public reporting of cardiac surgical outcomes. Recent reports in the national media have increased public awareness of this issue as it pertains to pediatric cardiac surgery in particular, and have stimulated further discussion of the role of public reporting, along with its goals, potential benefits, and unintended consequences. $^{2-4}$ The primary rationale for public reporting is to provide transparency and accountability, population.

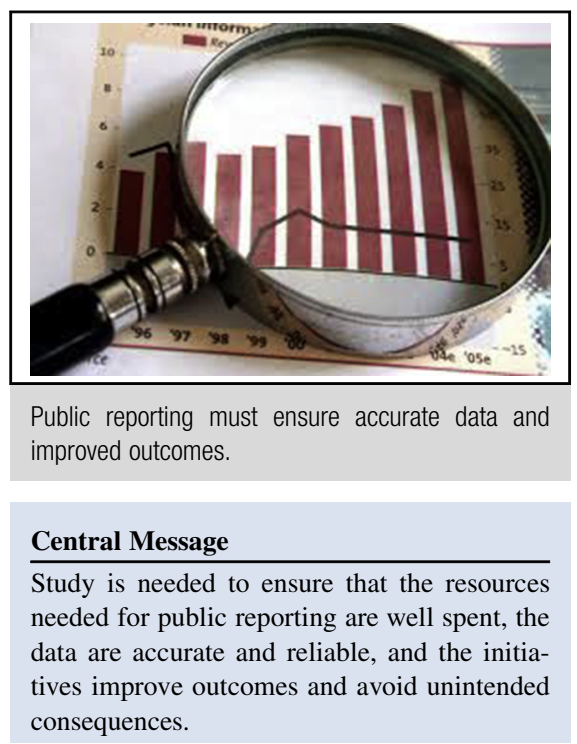

See Editorial Commentary page 908.

thus affirming the fundamental ethical right of patient autonomy. Additional goals of public reporting may include better education of healthcare consumers, reduced costs, improved resource utilization, and, most importantly, improved patient care and outcomes. Currently, public reporting in pediatric cardiac surgery is mandated in a handful of states, and the Society of Thoracic Surgeons (STS) recently initiated the first national reporting initiative, with approximately $60 \%$ of programs currently submitting data to the STS Congenital Heart Surgery Database. US News and World Report also publicly ranks and reports hospital performance on a variety of metrics in this

Although most would not argue that transparent sharing of information regarding care and outcomes with the public is not important, the impact of public reporting programs on patient outcomes is less clear. There is a paucity of data concerning the best designs of such programs to promote improvements in care and outcomes and avoid unintended consequences, including inadvertently steering patients toward a less-than-ideal hospital for their care, risk aversion among practitioners, and diversion of limited hospital resources from other, potentially more effective quality improvement efforts. ${ }^{5}$ These issues have received minimal 
attention in the pediatric population to date, but have been increasingly examined in adult cardiovascular medicine where public reporting programs have existed for longer periods. 5

In the March issue of the Journal of the American College of Cardiology, DeVore and colleagues evaluated the impact of public reporting by the Centers for Medicare \& Medicaid Services (CMS) on hospital readmission rates for acute myocardial infarction (AMI), heart failure, and pneumonia. ${ }^{1}$ They assessed trends in riskadjusted 30-day all-cause readmissions before and after implementation of public reporting by the CMS, and found that public reporting was not associated with any measurable change in readmission trends or allcause 30-day mortality rates for any of the conditions examined. ${ }^{1}$ They concluded that the 2009 CMS decision to publicly report readmission rates was not associated with improvements in the trends for readmission or postdischarge mortality. ${ }^{1}$

The study by DeVore and colleagues, along with other recent reports using robust statistical methodology and assessing outcomes adjusted for differences in case mix, add to a growing body of literature questioning whether improvements in patient outcomes through public reporting programs are achievable. ${ }^{2,5,6}$ The reasons for this are likely multifactorial and may include such factors as conflicting data on hospital performance reported by different agencies, issues with data quality and accuracy or analytic methodology such that reported data do not in fact reliably identify the highest- performing centers, and limited consumer understanding of reported metrics, among other factors. ${ }^{4-10}$ There can be considerable disagreement between national hospital ranking systems as to classification of high-performing and lowperforming hospitals. $^{11}$

As an example of some of these methodological issues, Krell and associates recently used data from the American College of Surgeons' National Surgical Quality Improvement Program to investigate the reliability of different outcome metrics (mortality, overall morbidity, and severe morbidity) commonly used for profiling hospital quality across several adult surgical procedures. ${ }^{12}$ Reliability is a measure of an outcome's capability to detect true differences in quality, if they exist. ${ }^{12}$ Reliability was generally low for most of the outcome metrics evaluated across procedures, related primarily to low case volume and infrequent outcomes. ${ }^{12}$ This finding is especially relevant to pediatric cardiac surgery, because case volumes for specific procedures are low and mortality for most procedures is rare. $^{7}$ Krell and colleagues noted that use of outcomes with low reliability can mask both poor and high performance compared with benchmark data. ${ }^{12}$ Several methodological approaches can optimize reliability, including aggregating individual procedures into larger groups, analyzing data over a longer time span, using composite quality metrics that integrate different aspects of quality and effectively increase the event rate, and using statistical methodologies related to "reliability adjustment"; however, all of these approaches may have certain limitations as well.

In addition, there are also an increasing number of reports questioning whether public reporting may be associated with unintended consequences, such as promoting risk aversion and limiting access to care. In a recent study evaluating the association of public reporting of outcomes for percutaneous coronary intervention (PCI) with management and outcomes for patients with AMI, Waldo and colleagues ${ }^{6}$ found that public reporting was associated with reduced use of PCI, particularly in patients with high-risk features, and worse outcomes overall in AMI patients, with increased in-hospital mortality (the opposite effect of what public reporting aims to achieve). Interestingly, the increase in AMI mortality occurred primarily in patients who did not receive PCI. ${ }^{6}$ This finding suggests the possibility of risk aversion and reduced use of PCI in high-risk patients, who some have argued may in fact have the most to gain from revascularization.

Similarly, in pediatric cardiac surgery, a recent poll of pediatric cardiac surgeons in the United Kingdom suggested that public reporting also may be associated with risk aversion in this population. ${ }^{13}$ Slightly less than one-third of surgeons in the anonymous poll reported recommending nonsurgical treatment because they feared the impact of mortality on their publicly reported outcomes. ${ }^{13}$ Although declining to take on a high-risk case at a low-volume or inexperienced center with referral to an experienced high-quality center may be a desired outcome of public reporting, denial of high-risk care altogether across the board owing to public reporting concerns would be an unintended consequence of such programs. ${ }^{2,3}$

It is clear that surgeons and physicians caring for children with congenital heart defects must be accountable for the outcomes of their interventions and management. In addition, patients and parents have the right to access information regarding care and outcomes to make the best decisions when choosing their providers. Public reporting is a tool to achieve these goals. To optimize these programs, further efforts are needed to better understand and design public reporting initiatives. In their recent article, DeVore and colleagues ${ }^{1}$ suggest that trials of quality improvement interventions such as public reporting are needed before 
widespread dissemination or utilization. Although a trial of public reporting would be infeasible owing to the ethical implications of withholding information from the public, there are several areas that may be studied further to improve our understanding and optimize public reporting programs.

First, for public reporting to achieve its intended goals, reported metrics must be accurate, able to reliably discern hospital quality, and avoid penalizing hospitals for taking on high-risk cases. In pediatric cardiac surgery, this can be particularly challenging owing to the very wide heterogeneity of disease and variability in case mix and volumes across centers. ${ }^{8-10,14}$ Ongoing efforts are geared toward defining the optimal population for public reporting initiatives (all operations vs more homogeneous subsets of procedures) and balancing competing goals of optimizing sample size/event rates and reducing heterogeneity. ${ }^{10}$ The methodology for case mix and patient characteristic adjustment has improved significantly in recent years, and further efforts are needed to continue to optimize current methods. ${ }^{15,16}$ Ongoing initiatives involving the development of composite metrics incorporating multiple domains of quality also may help address some of the concerns regarding reliability discussed above.

Second, reported metrics must be clearly reported and easy for stakeholders to interpret. This is critical if the data are to be used to steer patients toward higher-performing centers and/or to provide incentives for hospitals with lower performance to make improvements (both of which are likely necessary to drive improvements in overall care and outcomes). A recent survey of adults presented with cancer surgery data displayed in a fashion typical of surgical outcome reports showed that nearly one-half of those surveyed recommended switching surgeons, when the data displayed in fact did not indicate any clinically or statistically meaningful difference between the surgeons. ${ }^{17}$ This area remains understudied in pediatric cardiac surgery, although investigators from The Children's Hospital of Philadelphia have recently partnered with patient/parent advocacy groups to begin research in this area.

In addition, families must have the ability to choose a high-performing center, ideally based on geography and on the particular type and complexity of disease. This can be challenging, with some of the reporting methodologies used across various fields tending to classify most hospitals/providers in the average or middle categories, such that relatively few states may have a hospital classified as a "high performer," for example. Regional reporting or another methodology indicating where a hospital falls within larger categories may be useful for further investigation, as recommended by others. $^{18}$

Finally, it can be a challenge to understand a hospital's case mix or the type of patients that it serves relative to other hospitals. This is critical if reported metrics are to be interpreted correctly. Current statistical methodology, known as indirect standardization, used across virtually all public reporting programs to calculate "risk-adjusted" healthcare performance metrics allows assessment of a hospital's observed outcomes in relation to what would be expected if patients with a similar case mix had been cared for at an "average" hospital in the reference population. ${ }^{10,19,20}$ Because indirectly standardized outcomes are estimated only for the patients actually treated at a hospital, the results apply only to that hospital's particular case mix. A hospital can be classified as a high performer (performing better than what would be expected for its case mix; for example, receiving a 3-star STS designation) and achieve this through caring for a relatively highcomplexity population, a lower-complexity population, or somewhere in between. The results derived using indirect standardization cannot be used to directly compare 2 hospitals unless their case mix has been demonstrated to be similar, and it cannot be assumed that a hospital achieving better-than-average results in a generally low-risk population could do the same in a population of higher-risk patients. $^{10,19,20}$ Developing better methods to convey this information to consumers is vital. In addition, to promote the most appropriate interpretation and comparisons, it also is likely useful to consider reporting performance data stratified by a measure of case mix, as has been recommended by others. ${ }^{18}$ These strategies and others may help avoid the unintended situation of steering a patient with a high-complexity lesion to a center that is designated as a high performer because it achieved better-thanexpected outcomes in a lower-complexity patient population and has relatively little experience with highercomplexity patients.

The study by DeVore and associates is an example of the type of critical assessments that are needed to optimize our methods of public reporting. ${ }^{1}$ Further study is needed to ensure that the substantial resources needed to support these programs are well spent, that the public is provided with the most accurate and reliable data possible, and that public reporting initiatives have the greatest potential to improve outcomes while avoiding unintended consequences.

\section{Conflict of Interest Statement}

Authors have nothing to disclose with regard to commercial support. 


\section{References}

1. DeVore AD, Hammill BG, Hardy NC, Eapen ZJ, Peterson ED, Hernandez AF. Has public reporting of hospital readmission rates affected patient outcomes? Analysis of Medicare claims data. J Am Coll Cardiol. 2016;67:963-72

2. Pasquali SK, Dimick JB, Ohye RG. Time for a more unified approach to pediatric health care policy? The case of congenital heart care. JAMA. 2015;314:1689-90

3. Cohen E. After CNN investigation, a push to halt child heart surgery at some hospitals. Available at: http://www.cnn.com/2016/01/13/health/jama-childrensheart-surgery-hospitals/. Accessed November 21, 2016.

4. Ban KA, Cohen ME, Ko CY, Friedberg MW, Stulberg JJ, Zhou L, et al. Evaluation of the ProPublica Surgeon Scorecard "adjusted complication rate" measure specifications. Ann Surg. 2016;264:566-74.

5. Wasfy JH, Borden WB, Secemsky EA, McCabe JM, Yeh RW. Public reporting in cardiovascular medicine: accountability, unintended consequences, and promise for improvement. Circulation. 2015;131:1518-27.

6. Waldo SW, McCabe JM, O'Brien C, Kennedy KF, Joynt KE, Yeh RW. Association between public reporting of outcomes with procedural management and mortality for patients with acute myocardial infarction. J Am Coll Cardiol. 2015;65:1119-26

7. Jacobs JP, O'Brien SM, Pasquali SK, Jacobs ML, Lacour-Gayet FG, Tchervenkov CI, et al. Variation in outcomes for risk-stratified pediatric cardiac surgical operations: an analysis of the STS Congenital Heart Surgery Database. Ann Thorac Surg. 2012;94:564-71; discussion $571-2$.

8. Pasquali SK, Jacobs ML, O'Brien SM, He X, Gaynor JW, Gaies MG, et al. Impact of patient characteristics on hospital-level outcomes assessment in congenital heart surgery. Ann Thorac Surg. 2015;100:1071-6; discussion 1077.

9. Pasquali SK, Peterson ED, Jacobs JP, He X, Li JS, Jacobs ML, et al. Differential case ascertainment in clinical registry versus administrative data and impact on outcomes assessment for pediatric cardiac operations. Ann Thorac Surg. 2013; 95:197-203.

10. Pasquali SK, Wallace AS, Gaynor JW, Jacobs ML, O’Brien SM, Hill KD, et al. Congenital heart surgery case mix across North American centers and impact on performance assessment. Ann Thorac Surg. 2016;102: 1580-7.

11. Austin JM, Jha AK, Romano PS, Singer SJ, Vogus TJ, Wachter RM, et al National hospital ratings systems share few common scores and may generate confusion instead of clarity. Health Aff (Millwood). 2015;34:423-30.

12. Krell RW, Hozain A, Kao LS, Dimick JB. Reliability of risk-adjusted outcomes for profiling hospital surgical quality. JAMA Surg. 2014;149:467-74.

13. Knapton S. One in three heart surgeons refuse difficult operations to avoid poor mortality ratings. The Telegraph. June 3, 2016. Available at: http://www telegraph.co.uk/science/2016/06/03/one-in-three-heart-surgeons-refuse-difficultoperations-to-avoid/.

14. Jacobs JP, O’Brien SM, Pasquali SK, Kim S, Gaynor JW, Tchervenkov CI, et al The importance of patient-specific preoperative factors: an analysis of the Society of Thoracic Surgeons Congenital Heart Surgery Database. Ann Thorac Surg. 2014;98:1653-8; discussion 1658-9.

15. O'Brien SM, Jacobs JP, Pasquali SK, Gaynor JW, Karamlou T, Welke KF, et al. The Society of Thoracic Surgeons congenital heart surgery database mortality risk model: part 1-statistical methodology. Ann Thorac Surg. 2015;100:1054-62.

16. Jacobs JP, O’Brien SM, Pasquali SK, Gaynor JW, Mayer JE Jr, Karamlou T, et al The Society of Thoracic Surgeons congenital heart surgery database mortality risk model: part 2-clinical application. Ann Thorac Surg. 2015;100:1063-8; discussion 1068-70.

17. Weight CJ, Labine L, Palma I, Dixon C. Interpretation of an online surgeon scorecard by the general public and its effect on adults changing cancer surgeons even when presented with complication are statistically equivalent. J Clin Oncol. 2016;34(Suppl 2S):abstract 83.

18. Ash AS, Fienberg SE, Louis TA, Normand S-LT, Stukel TA, Utts J. Statistical issues in assessing hospital performance. Available at: https://www.cms.gov/ Medicare/Quality-Initiatives-Patient-Assessment-Instruments/HospitalQuality Inits/Downloads/Statistical-Issues-in-Assessing-Hospital-Performance.pdf. Accessed November 21, 2016.

19. Shahian DM, Normand S-LT. What is a performance outlier? BMJ Qual Saf 2015;24:95-9.

20. Shahian DM, Normand S-LT. Comparison of "risk-adjusted" hospital outcomes. Circulation. 2008;117:1955-63. 